\title{
Maturidade de gestão de projetos de sistemas de informação: um estudo exploratório quantitativo no Brasil
}

\author{
Renan Mastrange Guedes ${ }^{\mathrm{a}}$, Marilson Alves Gonçalves (in memoriam), \\ Fernando José Barbin Laurindo $^{c}$, Antonio Cesar Amaru Maximiano ${ }^{d}$ \\ a*renan.guedes@usp.br, FEA/USP, Brasil \\ bmarilson@usp.br, FEA/USP, Brasil \\ ‘fjblau@usp.br, Poli/USP, Brasil \\ dmaximin@usp.br, FEA/USP, Brasil
}

\begin{abstract}
Resumo
Este artigo é resultado de uma pesquisa cujo objetivo foi identificar diferenças na maturidade de gestão de projetos de sistemas de informações em tecnologia da informação ( $\mathrm{Tl} / \mathrm{Sl}$ ) nas diferentes organizações. Foram aplicados questionários eletrônicos para mensurar a percepção de profissionais sobre a maturidade dos projetos de $\mathrm{Tl} / \mathrm{Sl}$ em sua organização. Compreende a análise de 56 organizações com atuação no Brasil, envolvendo os setores de serviços, comercial e público, inclusive o terceiro setor. A pesquisa se desenvolveu durante o ano de 2011 e foi apoiada por um framework conceitual construído com base em vários trabalhos empíricos sobre o tema, principalmente o 0 PM $3^{\circledR}$. 0 artigo apresenta resultados que evidenciam que a maturidade de gestão de projetos de Tl/SI das organizações não apresenta diferença significativa entre os setores. No entanto, aponta evidências de que grandes organizações possuem maior maturidade em gestão de projetos Tl/S1.
\end{abstract}

Palavras-chave

Gestão de projetos. Tecnologia da informação. Sistemas de informação. Maturidade de gestão de projetos. Projetos de tecnologia da informação.

\section{Introdução}

Diversos pesquisadores procuraram aplicar os modelos de maturidade de gestão de projetos em estudos empíricos, investigando possíveis diferenças de maturidade de gestão de projetos entre diversos setores de atividade (BAl et al., 2010; BYDE, 2008; COOKE-DAVIES; ARZYMANOW, 2003; CRAWFORD, 2005; MULLALY, 2006; GUANGSHE et al., 2008; IBBS; REGINATTO; KWAK, 2004; LEVENE; BENTLEY; JARVIS, 1995; GRANT; PENNYPACKER, 2006; SILVEIRA, 2008; ZWIKAEL; GLOBERSON, 2006).

Este estudo se propõe a analisar a maturidade de gestão de projetos de sistemas de informação ou, como mais recentemente vêm sendo chamados, projetos de sistemas de informações em tecnologia da informação ( $\mathrm{Tl} / \mathrm{SI})$, admitindo que seria pouco apropriado avaliar as diferenças de maturidade de gestão de projetos entre diversos setores. Pela análise, considerou-se também todos os tipos de projeto que as organizações executam, apoiando-se na tipologia proposta por Archibald (2004).

Decorrente da hipótese de que, mesmo dentro da mesma organização, projetos de mudança organizacional ou negócios possuem maturidade diferente da encontrada em projetos de desenvolvimento de produtos e serviços, ou em projetos de pesquisa e desenvolvimento ou em projetos de sistemas de informação (softwares).

A importância da gestão de projetos de Tl para o meio acadêmico pode ser evidenciada tomando-se por base, por exemplo, o Project Management Journal e seu predecessor o Project Management Quartely, ambas revistas acadêmicas do PMI (Project Management Institute). 
Segundo Rivard e Dupré (2009), dos aproximadamente 700 artigos publicados entre 1970 e 2005 nessas revistas, 40 abordavam a gestão de projetos de sistemas de informação. Os autores ainda apontaram que entre os anos de 2000 e 2004 mais de $15 \%$ dos artigos publicados que versaram sobre essa temática evidenciam a importância recente do tema.

Acrescenta-se a isto o potencial dos projetos de $\mathrm{Tl}$ em facilitar e promover mudanças estratégicas, organizacionais e operacionais nas organizações (LAURINDO, 2008).

\section{Revisão bibliográfica}

Segundo Archibald (2004), os projetos podem ser classificados em categorias (Quadro 1). Ele ainda sugere que as características particulares de cada categoria sejam estudadas de maneira independente. Dessa forma, cada categoria de projeto poderia ser investigada de maneira mais apropriada, considerando-se suas nuances e especificidades (CRAWFORD; HOBBS; TURNER, 2004; CRAWFORD, 2005; SHENHAR; WIDEMAN, 1996; YOUKER, 1999).

Rivard e Dupré (2009) apontam importantes tendências na pesquisa em gestão de projetos de Tl, sendo a primeira delas a de enriquecer as melhores práticas, exemplificadas de maneira especial pelo $\mathrm{PMBOK}^{\circledR}$ com processos, ferramentas e técnicas de gestão de stakeholders como usuários de sistemas. Outra tendência importante apontada por Rivard e Dupré (2009) e por Luftman e Kempaiah (2007) está associada à crescente tendência de terceirização

Quadro 1. Categoria de projetos.

\begin{tabular}{|c|c|}
\hline Categorias & Subcategorias \\
\hline 1. Projetos de Defesa, Segurança e Aeroespaciais & $\begin{array}{l}\text { 1.1 Sistemas de defesa } \\
\text { 1.2 Espacial } \\
\text { 1.3 Operações militares }\end{array}$ \\
\hline 2. Projetos de Mudanças Organizacionais e em Negócios & $\begin{array}{l}\text { 2.1 Aquisição/Fusão } \\
\text { 2.2 Melhoria de processos de gestão } \\
\text { 2.3 Empreendimento de novos negócios } \\
\text { 2.4 Reestruturação organizacional } \\
\text { 2.5 Eventos judiciais } \\
\text { 2.6 Mudanças organizacionais derivadas de Qualidade Total }\end{array}$ \\
\hline $\begin{array}{l}\text { 3. Projetos de Sistemas de Comunicação (dados, voz e } \\
\text { imagem) }\end{array}$ & $\begin{array}{l}\text { 3.1 Sistemas de comunicação em rede } \\
\text { 3.2 Sistemas de comunicação switching }\end{array}$ \\
\hline 4. Projetos de Eventos & $\begin{array}{l}\text { 4.1 Eventos internacionais } \\
\text { 4.2 Eventos nacionais }\end{array}$ \\
\hline $\begin{array}{l}\text { 5a. Projetos de Construção Leve (design de engenharia, } \\
\text { arquitetura etc.) }\end{array}$ & 5a.1 Engenharia, arquitetura, decoração e afins \\
\hline $\begin{array}{l}\text { 5b. Projetos de Construção Pesada (empreendimentos, } \\
\text { investimentos, construções e obras) }\end{array}$ & $\begin{array}{l}\text { 5b.1 Desmontagem } \\
\text { 5b.2 Demolição } \\
\text { 5b.3 Manutenção e modificação } \\
\text { 5b.4 Projeto/contratação/construção (civil, energia, meio ambiente, } \\
\text { edificações, industrial comercial, residencial, naval) }\end{array}$ \\
\hline 6. Projetos de Sistemas de Informação (softwares) & - Desenvolvimentos de novo sistemas de informação (Tl) \\
\hline 7. Projetos de Desenvolvimento Regional ou Internacional & $\begin{array}{l}\text { 7.1 Desenvolvimento agropecuário/rural } \\
\text { 7.2 Educação } \\
\text { 7.3 Saúde } \\
\text { 7.4 Nutrição } \\
\text { 7.5 Populacional } \\
\text { 7.6 Empreendimentos em pequena escala } \\
\text { 7.7 Infraestrutura: energia (petróleo, gás, carvão, geração e distribuição } \\
\text { de energia, industrial, telecomunicações, transportes, urbanização, } \\
\text { fornecimento e tratamento de água, irrigação) }\end{array}$ \\
\hline 8. Projetos de Entretenimento e Mídia & $\begin{array}{l}\text { 8.1 Filme } \\
\text { 8.2 Programa de TV } \\
\text { 8.3 Peça teatral ou apresentação musical }\end{array}$ \\
\hline 9. Projetos de Desenvolvimento de Produtos ou Serviços & $\begin{array}{l}\text { 9.1 Equipamentos de informática } \\
\text { 9.2 Produtos/processos industriais } \\
\text { 9.3 Produtos/processos para o consumo } \\
\text { 9.4 Produtos/processos farmacêuticos } \\
\text { 9.5 Serviços (financeiros, outros) } \\
\end{array}$ \\
\hline 10. Projetos de Pesquisa e Desenvolvimento & $\begin{array}{l}\text { 10.1 Meio ambiente } \\
\text { 10.2 Industrial } \\
\text { 10.3 Desenvolvimento econômico } \\
\text { 10.4 Medicina } \\
\text { 10.5 Científico }\end{array}$ \\
\hline
\end{tabular}

Fonte: adaptado de Archibald (2004). 
(outsourcing) no ambiente de projetos. Assim sendo, seria louvável ampliar a discussão no meio acadêmico e profissional em relação a esse fenômeno e a seu impacto na gestão de projetos.

Em um estudo sobre a taxa de sucesso de projetos de Tl, Jeffery e Leliveld (2004) estimaram que 68\% dos projetos corporativos de $\mathrm{Tl}$ não alcançam os objetivos de negócio originais e excedem o prazo e orçamento previstos. Posteriormente, Standish Group International (2009) estimou que 44\% dos projetos de $\mathrm{Tl}$ entre 2008 e 2009 nos Estados Unidos estavam atrasados ou foram entregues com atraso, além de terem excedido o orçamento, e seus resultados não atenderam às especificações. Os autores estimam ainda que $24 \%$ dos projetos em questão geraram produtos finais que não foram utilizados ou mesmo foram cancelados antes de terminarem.

McFarlan (1981) já havia apresentado estatísticas alarmantes em relação ao sucesso dos projetos de Tl, tendo apontado como uma das possíveis origens do insucesso a incapacidade de avaliar adequadamente 0 risco individual dos projetos ou mesmo de consolidar essa análise em nível de portfólio de projeto.

Mais recentemente, Flyvbjerg e Budzier (2011) investigaram 1.471 projetos de $\mathrm{Tl}$, comparando orçamentos e retornos estimados com os custos e resultados alcançados e encontraram um estouro orçamentário médio de $27 \%$. Entretanto, ao ilustrar graficamente a distribuição da variação de custos, os pesquisadores encontram uma cauda longa que aponta que alguns projetos, em específico, apresentam distância bastante alta da média e do desvio-padrão. Os autores ainda identificaram que um a cada seis projetos da amostra estoura o orçamento em $200 \%$, na média, e os prazos são excedidos em aproximadamente $70 \%$ deles. Esses projetos foram denominados cisnes negros por estarem na cauda longa da distribuição.

Não obstante os estudos de Flyvbjerg e Budzier (2011) e McFarlan (1981) terem aproximadamente 30 anos de distância um do outro, ambos sugerem a mesma falha recorrente em projetos de Tl: a dificuldade de avaliar adequadamente os riscos de cada projeto e de balanceá-los em nível de portfólio.

Dessa forma, parece existir certo espaço para o desenvolvimento de estudos que busquem auxiliar de alguma forma as organizações a melhorarem a taxa de sucesso e o retorno sobre portfolio de projetos de $\mathrm{Tl}$.

\subsection{Maturidade e capacidade de processos e governança de $T 1$}

Maturidade, do latim maturitate, é o mesmo que madureza, idade madura ou perfeição. Pode ser entendida como o grau em que as atitudes, a socialização e a estabilidade afetiva de um indivíduo refletem, como característica normal do homem adulto, um estado de adaptação ou ajustamento ao seu próprio meio (MICHAELIS, 1998).

Michaelis (1998) atesta que capacidade, derivada do latim capacitate, seria o mesmo que o poder, aptidão ou possibilidade de fazer ou produzir qualquer coisa. Por sua vez, para a Psicologia, capacidade seria a potencialidade individual para o exercício de qualquer função, a qual encontra limite na constituição psicofisiológica. Para a administração de operações, os conceitos de maturidade e de capacidade remetem ao controle estatístico do processo (SPC, do inglês, Statistical Process Control), em que a aplicação de suas técnicas leva a dois resultados básicos: 1) redução da variabilidade inerente ao processo; e 2) melhoria na eficiência e eficácia do processo (DEMING, 1986; COOKE-DAVIES; ARZYMANOW, 2003; SALVIANO; FIGUEIRED0, 2008).

Dessa forma, por mero silogismo, pode-se induzir que quanto maior a maturidade, maior seria a capacidade, usando-se como base o sentido etimológico das palavras. Assim, Lee e Anderson (2006) propõem uma correlação positiva entre a maturidade em gestão de projetos de Tl e a capacidade de gestão de projetos de $\mathrm{Tl}$, sendo que se deve levar em consideração também alguns fatores adicionais exógenos e endógenos.

0 surgimento dos modelos de maturidade de gestão de projetos, por sua vez, é comumente relacionado aos modelos de maturidade de processo de Tl, em especial, o CMM (Capability Maturity Model), modelo este desenvolvido pelo SEl (Software Engineering Institute) da Univesidade Carnagie-Mellon entre 1986 e 1993 (GRANT; PENNYPACKER, 2006; COOKE-DAVIES; ARZYMANOW, 2003; RODRIGUES; RABECHINI JUNIOR; CSILLAG, 2006; JUGDEV; THOMAS, 2002; KWAK; IBBS, 2000a, b; KERZNER, 2001).

Originalmente, alguns autores como Smith, Mitchell e Summer (1985), Scott (1971), Chandler (1962) e Haire (1959) buscaram conceituar maturidade associada a processo ou, em inglês, process capability. Esses trabalhos sugeriam que as organizações amadurecem por estágios, seguindo um determinado padrão uniforme.

Embora essa uniformidade possa ser questionada, os modelos de avaliação de maturidade por estágios se tornaram ferramentas comuns para a padronização de processo, como as ISO 9000, 9001 e demais da International Organization for Standarization, para a engenharia de software com o CMM (Capability Maturity Model) e o CMMI (Capability Maturity Model Integration) da SEI (Software Engineering Institute) 
e, também, para a gestão de projetos com o PMBOK Guide (Project Management Body of Knowledge Guide).

Segundo Von Wangenheim et al. (2010), o modelo mais utilizado nas pesquisas empíricas sobre maturidade em $\mathrm{Tl}$ seria o CMM, que é aplicado em 58\% das pesquisas avaliadas, seguido pelo 1SO/IEC 15504, que é usado em 36\% dos estudos. Já os demais modelos seriam o CMMl, o ISO 9000 e o ISO/IEC 12207, que aparecem, respectivamente, em 21\%, 17\% e 15\% das pesquisas.

Harter, Krishnan e Slaughter (2000) investigaram os efeitos da maturidade sobre a qualidade, o tempo de ciclo e o esforço em projetos de desenvolvimento de software. Esses pesquisadores encontraram evidências de que aumentar o nível de maturidade dos projetos pode levar a uma maior qualidade, no entanto esse incremento de maturidade aumenta o esforço. Esses autores apontam ainda que aumentar a qualidade pode levar à redução do tempo de ciclo e, assim, reduzir o esforço.

Foi em meio a esse contexto que se iniciou a discussão sobre maturidade de gestão de projetos tanto no meio profissional quanto no meio acadêmico. Em 1998, o PMl (Project Management Institute) desenvolveu, com o objetivo de estabelecer um padrão para os modelos de maturidade em gestão de projetos, o OPM3 (Organizational Project Management Maturity Model) (GRANT; PENNYPACKER, 2006).

A maturidade de gestão de projetos acabou transcendendo somente o escopo dos processos de gestão, visto que os modelos que têm sido discutidos avaliam também os indivíduos em relação a suas competências, a equipe e os stakeholders no que se refere a suas relações, a organização em relação a seus processos, sistemas, cultura e estrutura e, finalmente, o contexto ou ambiente competitivo (FRAME, 1999; RABECHINI JUNIOR, 2003; RODRIGUES; RABECHINI JUNIOR; CSILLAG, 2006).

Embora os modelos de maturidade de gestão de projetos sejam criticados por Jugdev e Thomas (2002) por motivos diversos, alguns trabalhos empíricos constataram evidências de que maior maturidade em gestão de projetos poderia provocar melhor desempenho organizacional (BERSSANETI et al., 2008; COOKE-DAVIES; ARZYMANOW, 2003; DORLING, 1993; KWAK; IBBS, 2000a, b; MORAES; KRUGLIANSKAS, 2010).

A aplicação de um modelo de maturidade ou outro não gera por si só vantagem competitiva e, em alguns casos, pode-se até mesmo entender que o uso de padrões leva a convergência competitiva, visto que as práticas de negócio passam a ser bastante semelhantes de uma organização para outra no longo prazo (BARNEY; HESTERLY, 1986; PORTER,
1980). Segundo Collins (2001, 2009) e Collins e Hansen (2011), o que cria vantagem competitiva ao longo do tempo não é certa prática ou modelo que a organização escolhe utilizar. Conforme o autor, a vantagem competitiva sustentável é resultado de como a organização faz uso de certa prática e por qual motivo ela a aplica. 0 autor ainda reforça a importância de a organização compreender sob quais circunstâncias e em quais contextos tal prática não seria mais válida.

A incapacidade de reconhecer que projetos diferentes exigem abordagens distintas é, conforme McFarlan (1981), uma possível falha na gestão de projetos de Tl. Esse autor propõe que se apliquem as ferramentas de gestão de projetos de maneira geral por quatro métodos distintos: integração externa, integração interna, planejamento formal e controle formal. Ele ainda complementa, identificando oito tipos de projetos de $\mathrm{Tl}$, considerando-se o nível de estrutura, tecnologia e tamanho, o que antecipa algumas das dimensões posteriormente empregadas por Shenhar e Dvir (2010).

Shenhar e Dvir (2010) enfatizam a necessidade de buscar-se aplicar processos e técnicas de gestão distintas em projetos que tenham diferentes composições de complexidade, ritmo, inovação e tecnologia. Assim, as quatro dimensões do diamante, como os autores as intitulam, provocam uma necessidade de adaptação às práticas de gestão de projetos.

Como cada projeto é único, poderia se dizer que o conjunto de processos e práticas para seu sucesso pode ser variável, seja devido às dimensões de Shenhar e Dvir (2010), seja devido aos quatros métodos gerais de gestão apontados por McFarlan (1981) em projetos de Tl. Dessa maneira, Shenhar e Dvir (2010) e McFarlan (1981) confirmam o entendimento de Collins (2001, 2009) e Collins e Hansen (2011) de que é necessário compreender-se sob quais circunstâncias e em quais contextos algumas práticas beneficiam a organização e quando elas não são válidas.

Moore $(2002,2004)$ analisa o desafio de se buscar a inovação contínua do negócio em torno de processos-chave de uma organização, denominados de core em seu trabalho. Segundo o autor, o core é o que diferencia as organizações aos olhos do cliente, enquanto todo o restante dos processos são somente commodities ou, como denominado por ele, contexto. A finalidade do core de uma empresa é direcionar a inovação do negócio e buscar diferenciação em relação ao competidor para estimular o crescimento.

0 autor ainda aponta que o objetivo central dos processos de contexto é serem operados da maneira mais eficiente possível. Assim, os processos somente permanecem como core enquanto ainda 
geram vantagem competitiva. Uma vez copiado, o core se transforma em contexto e já não é mais uma inovação. Nesse ponto, a organização deve reformular o processo contexto, buscando padronização, eficiência e a produtividade.

Entende-se, assim, que somente é commodity aquele processo de negócio que não traz vantagem competitiva, que é relativamente padronizado na indústria e que não é fonte de diferenciação. Portanto, ao perseguir um papel estratégico, a Tl poderia se posicionar na busca de inovação e vantagem competitiva, gerenciando a camada "comoditizada" com foco em disponibilidade e reformulando os processos de contexto para possam voltar a trazer algum tipo de vantagem competitiva (BARNEY; HESTERLY, 1986; GUEDES et al., 2011; JUGDEV; THOMAS, 2002; MOORE, 2004; PRAHALAD; HAMEL, 1990).

Assim, a teoria parece sugerir que os modelos de maturidade podem auxiliar na construção de ativos estratégicos ou competências chave, levando assim à criação, manutenção e renovação de vantagem competitiva, caso esses modelos sejam utilizados como um meio para tornar os processos mais eficientes e não como um fim em si mesmos.

\subsubsection{Modelos de maturidade de gestão de projetos}

A análise dos modelos de maturidade de gestão de projetos considerados neste trabalho não tem por objetivo exaurir o tema. Seu enfoque central é proporcionar uma plataforma teórica com base nos modelos mais comumente utilizados no mundo acadêmico e corporativo no Brasil. Assim, foi dada maior ênfase ao modelo que será mais focado nesta pesquisa.

Segundo Silveira (2008), os modelos mais conhecidos de maturidade em gerenciamento de projetos seriam:

- Project Management Maturity Model (PMMM), da PM Solutions;

- Kerzner Project Management Maturity Model (KPMMM);

- ESI International Project Framework (ESI);

- PM3 - Project Management Maturity Model (Berkeley);

- Project Management Process Maturity (PM) ${ }^{2}$;

- OGC Project Management Process Maturity;

- Integrated Management Systems Incorporated (IMSI);

- OGC Portfolio Management Maturity Model (P3M3);

- $O P M 3^{\circledR}$ - Organizational Project Management Maturity Model $\left(\mathrm{PMI}^{\circledR}\right)$.
Além dos modelos internacionais de maturidade em gestão de projetos indicados por Silveira (2008), há duas propostas de modelos nacionais que interessam a este trabalho de pesquisa, uma vez que o escopo deste artigo é a avaliação de maturidade da gestão de projetos em organizações no Brasil. São estes o modelo de Maximiano e Rabechini (2002) e o modelo de maturidade em gerenciamento de projetos (MMGP) de Prado (2008a).

Como será discutido adiante, este trabalho será baseado principalmente no OPM3 que, por essa razão, é apresentado em um nível maior de detalhes que os demais modelos.

\subsubsection{Organizational Project Maturity Management Model (OPM3)}

A criação do modelo Organizational Project Maturity Management Model levou em consideração o conceito Quality Function Deployment (QFD), em específico a abordagem house of quality, que utiliza matrizes para organizar e relacionar dados.

A função básica do modelo é auxiliar as organizações a entenderem e avaliarem o seu nível de maturidade em gerenciamento de projetos. Através do reconhecimento das competências que devem ser desenvolvidas, o modelo prevê um caminho para o melhoramento do gerenciamento de projetos da empresa (LUKOSEVICIUS, 2005).

Sendo assim, a segunda edição do $0 \mathrm{PM} 3^{\circledR}$ foi reconhecida pela American National Standards Institute (ANSI) como um American National Standard (ANSI/PMI 08-004-2008).

0 OPM3 é composto pelas melhores práticas de processos, capacidades que são as competências específicas necessárias à gestão de projetos, resultados tangiveis ou intangíveis que comprovam a existências das capacidades e, finalmente, indicadores de desempenho (RODRIGUES; RABECHINI JUNIOR; CSILLAG, 2006; PROJECT..., 2008).

Assim, o $\mathrm{OPM} 3^{\circledast}$ se apresenta como uma proposta de um processo de melhoria contínua semelhante aos ciclos de PDCA e de DMAIC composto de um conjunto formado pelos seguintes passos: (1) preparar o levantamento; (2) realizar o levantamento; (3) planejar melhorias; (4) implementar melhorias; e (5) repetir o processo (PROJECT..., 2008).

Segundo Silva Junior (2009), o OPM ${ }^{\circledR}$ trabalha com quatro domínios definidos: organizacional, portfólio, programas e projetos. Assim, o modelo inova ao trazer uma característica importante, que é a avaliação não só da maturidade em gestão de projetos, mas também em gestão de portfólio, programas e projetos, bem como dos elementos organizacionais 
e, algumas vezes, culturais que reforçam e exercem influência sobre a orientação a projetos.

\subsection{Análise crítica dos modelos de maturidade de gestão de projetos}

Cooper, Edgett e Kleinschmidt (2001a, b) argumentam que há duas maneiras de uma empresa ser bem-sucedida em relação a sua carteira de projetos: realizar os projetos corretamente e realizar os projetos corretos. Assim, aumentar a maturidade de gestão de projetos pode ajudar na realização correta dos projetos.

A gestão de projetos sofre influência de vários fatores, como os relativos ao contexto tecnológico no qual a organização se encontra, ao porte da empresa e à experiência profissional do seu grupo de gestão, o que justifica a elaboração de estudos exploratórios para identificar os efeitos desses aspectos na maturidade ou mesmo para justificá-la e aprimorar sua utilização.

Os primeiros estudiosos propuseram modelos unidimensionais que avaliam a maturidade de gestão de projetos de maneira geral e nos quais a organização amadurece por estágios seguindo certa sequência ou padrão (KERZNER, 2001; MAXIMIANO; RABECHINI, 2002).

0 modelo KPMMM de Kerzner (2001) e o modelo de Maximiano e Rabechini (2002) são propostas de avaliação geral da maturidade de gestão de projetos em uma única dimensão, o que induz ao entendimento de que a organização amadurece por estágios relativamente definidos e segue certa sequência ou padrão, o que parece ser uma simplificação, talvez exagerada, da realidade.

Já outros autores sugerem modelos multidimensionais que avaliam a maturidade de gestão de projetos sob algumas características específicas, onde a organização não amadurece por estágios bem definidos e cada dimensão é relativamente independente, apontando também a existência de facilitadores organizacionais, ou seja, ativos intangiveis que ajudam a promover a prática de gestão de projetos (PRADO, 2006; PROJECT..., 2008).

0 MMGP de Prado (2006) avalia a maturidade de gestão de projetos em seis dimensões distintas que estão mais associadas somente aos facilitadores organizacionais da gestão de projetos. No entanto, esse modelo não analisa a maturidade de processos de gestão de projetos propriamente ditos.

Já o de PMI (PROJECT..., 2008) avalia tanto a maturidade dos processos de gestão de projetos de Kerzner (2001) e de Maximiano e Rabechini (2002) quanto às características facilitadoras, como vistas em Prado (2006), como avaliam também a maturidade dos processos de gestão de portfólio e programas.
0 OPM $3^{\circledR}$ supõe níveis distintos de maturidade em gestão de portfólio, programas e projetos, bem como de grupo de processo e dos facilitadores organizacionais. Essa flexibilidade parece evitar uma possível simplificação da realidade.

Segundo Silveira (2008), o modelo OPM3 ${ }^{\circledR}$ é uma combinação do mais compreensivo conjunto de melhores práticas organizado por meio de algumas dimensões. Esse estudo explora as dimensões organizacionais e de gestão de projetos, excluindo as dimensões de programas e portfólio.

Diferentemente de outros modelos, o OPM $3^{\circledR}$ classifica a maturidade em níveis em uma escala, o que beneficia a empresa no sentindo de medir e trabalhar nas práticas que trouxerem maiores benefícios estratégicos em vez de trabalhar nas práticas exclusivas de um nível (KANUP, 2009).

Outro fator importante é que ele também introduz um olhar mais abrangente, incluindo a avaliação dos programas e portfólios, pois enquanto o projeto é um esforço para criar um produto ou serviço, o programa é um conjunto de projetos relacionados e o portfólio é um conjunto de projetos ou programas agrupados com outros trabalhos, o que visa facilitar o gerenciamento dos trabalhos a fim de atender aos objetivos estratégicos da organização (SILVA JUNIOR, 2009).

Assim, este estudo focou na aplicação do modelo de maturidade organizacional de gestão de projetos $0 \mathrm{PM} 3^{\circledR}$, mas não se pretende assim exaurir o assunto ou mesmo identificar o melhor ou pior modelo de maturidade em gerenciamento de projetos quanto ao melhor desempenho das organizações.

No entanto, seria importante a consolidação de uma metodologia e instrumento padrão para avaliação da maturidade da gestão de projetos, para que possam ser realizadas comparações de resultados mais robustas entre as diversas pesquisas realizadas, visando conclusões também mais robustas e análises mais aprofundadas.

A escolha do $0 \mathrm{PM} 3^{\circledR}$ se deu pelo entendimento gerado pela revisão bibliográfica de que esse instrumento incorpora os elementos-chave de avaliação da maturidade em gestão de projetos necessários a este trabalho. Também é possível inferir pelo número de membros, certificados e chapters do $\mathrm{PMI}^{\circledast}$ no Brasil que ele será em breve o padrão mais difundido.

Entretanto, considerando os modelos nacionais Prado (2006, 2008a, b) e Maximiano e Rabechini (2002), notamos uma particularidade no $0 P M 3^{\circledR}$ e mesmo em outros modelos internacionais. Os modelos de avaliação de maturidade em gestão de projetos nacionais estudados (PRADO, 2006, 2008a, b; MAXIMIANO; RABECHINI, 2002) apontam a existência de um grau embrionário ou preliminar de maturidade. 


\subsection{Estudos empíricos de maturidade de gestão de projetos}

Durante a pesquisa bibliográfica foram identificados diversos artigos acadêmicos que descrevem, comparam e até mesmo propõem modelos de medição da maturidade. No entanto, poucos são os trabalhos empíricos disponíveis nas bases de dados Academic Search Premier (EBSCO), Web of Science (ISI), ABI/ INFORM (ProQuest) ou mesmo nas revistas acadêmicas (Project Management Journal - PMI - e International Project Management Journal - IPMA/Elsevier) que se propõem a realizar análise cruzada entre setores. Também não foram encontrados muitos estudos relevantes que procurassem investigar as correlações entre as características das organizações a e maturidade da gestão de projetos.

Ainda durante o levantamento de estudos anteriores não foram encontrados nessas bases e revistas trabalhos cuja proposta fosse realizar uma análise comparativa da maturidade da gestão de projetos para projetos de $\mathrm{Tl}$ entre setores de atividade distintos e que levassem em consideração o porte ou a amplitude geográfica de atuação da organização.

0 Quadro 2 consolida de forma não exaustiva os trabalhos empíricos mais recentes que procuraram estudar o problema da maturidade da gestão de projetos. Embora tais estudos não sejam semelhantes a esta pesquisa em termos de contexto temporal e geográfico, na metodologia e instrumentos de

Quadro 2. Estudos de avaliação da maturidade da gestão de projetos.

\begin{tabular}{|c|c|c|c|}
\hline Objetivo de pesquisa & Autores & Metodologia & Conclusões \\
\hline $\begin{array}{l}\text { ldentificar competências que geram a } \\
\text { maturidade na gestão de projetos. }\end{array}$ & $\begin{array}{l}\text { Levene, Bentley e } \\
\text { Jarvis (1995) }\end{array}$ & $\begin{array}{l}\text { Estudo em } 13 \text { empresas que } \\
\text { utilizam gestão de projetos de } \\
\text { tecnologia de informação. }\end{array}$ & $\begin{array}{l}\text { Maturidade na gestão de projetos } \\
\text { de tecnologia de informação, } \\
\text { entre empresas de setores diversos } \\
\text { (telecomunicação, bancário e de } \\
\text { prestação de serviços essenciais) são } \\
\text { similares. }\end{array}$ \\
\hline $\begin{array}{l}\text { ldentificar impactos financeiros e } \\
\text { organizacionais da gestão de projetos. }\end{array}$ & Kwak e lbbs (2000a) & $\begin{array}{l}\text { Questionário de } 148 \text { questões } \\
\text { efetuado em } 38 \text { empresas de } 4 \\
\text { setores diferentes. }\end{array}$ & $\begin{array}{l}\text { Empresas de engenharia }(3,36) \\
\text { e manufatura de alta tecnologia } \\
(3,34) \text { possuem maiores níveis } \\
\text { de maturidade, se comparadas a } \\
\text { empresas de telecomunicações }(3,30) \\
\text { e tecnologia de informação }(3,06) \text {. }\end{array}$ \\
\hline $\begin{array}{l}\text { ldentificar a natureza e a extensão das } \\
\text { diferenças de maturidade na gestão de } \\
\text { projetos entre empresas de seis setores } \\
\text { diferentes. }\end{array}$ & $\begin{array}{l}\text { Cooke-Davies e } \\
\text { Arzymanow (2003) }\end{array}$ & Estudo qualitativo em 27 empresas. & $\begin{array}{l}\text { Empresas do setor petroquímico } \\
\text { possuem uma maior maturidade na } \\
\text { gestão de projetos, se comparadas ao } \\
\text { setor de defesa e farmacêutico. }\end{array}$ \\
\hline $\begin{array}{l}\text { Medir a maturidade na gestão de } \\
\text { projetos identificando fatores de } \\
\text { influência. }\end{array}$ & $\begin{array}{l}\text { Grant e Pennypacker } \\
\text { (2006) }\end{array}$ & $\begin{array}{l}\text { Survey com } 123 \text { empresas } \\
\text { (manufatura, informação, finanças e } \\
\text { serviços técnicos profissionais). }\end{array}$ & $\begin{array}{l}\text { Grande maioria das empresas ( } 67 \% \text { ) } \\
\text { possuem níveis baixos de maturidade. } \\
\text { Não foram verificadas diferenças } \\
\text { estatisticamente relevantes entre } \\
\text { empresas de setores diversos e } \\
\text { empresas de tamanhos diversos. }\end{array}$ \\
\hline $\begin{array}{l}\text { Avaliar a influência do uso das } \\
\text { metodologias de gestão de projetos na } \\
\text { performance organizacional. }\end{array}$ & Crawford (2005) & $\begin{array}{l}\text { Survey envolvendo } 208 \\
\text { respondentes em } 3 \text { países (Estados } \\
\text { Unidos, Reino Unido e Austrália). }\end{array}$ & $\begin{array}{l}\text { Não há correlação estatística, avaliada } \\
\text { de forma geral e entre os elementos } \\
\text { da metodologia. }\end{array}$ \\
\hline $\begin{array}{l}\text { Acompanhamento dos níveis de } \\
\text { maturidade da gestão de projetos em } \\
\text { setores diversos ao longo do tempo. }\end{array}$ & Mullaly (2006) & $\begin{array}{l}\text { Survey com } 2.500 \text { participantes de } \\
550 \text { empresas do Canadá e Estados } \\
\text { Unidos, ao longo de } 6 \text { anos ( } 1998 \\
\text { a 2003). }\end{array}$ & $\begin{array}{l}\text { Verificado declínio nos níveis de } \\
\text { maturidade ao longo do tempo, } \\
\text { principalmente no setor de } \\
\text { telecomunicação, motivado pela } \\
\text { desregulamentação. }\end{array}$ \\
\hline $\begin{array}{l}\text { ldentificar os setores que possuem } \\
\text { maior qualidade de planejamento e } \\
\text { execução de projetos. }\end{array}$ & $\begin{array}{l}\text { Zwikael e Globerson } \\
\qquad(2006)\end{array}$ & $\begin{array}{l}\text { Survey aplicado com } 282 \text { gestores } \\
\text { de projetos. }\end{array}$ & $\begin{array}{l}\text { Maiores níveis de maturidade em } \\
\text { empresas de construção e engenharia } \\
(3,6) \text { se comparadas a empresas de } \\
\text { outros setores: software }(3,4) \text {, serviços } \\
(3,3) \text { e manufatura }(3,0) \text {. }\end{array}$ \\
\hline $\begin{array}{l}\text { Verificar se os setores econômicos } \\
\text { relacionados à construção possuem } \\
\text { maiores níveis de maturidade na } \\
\text { gestão de projetos. }\end{array}$ & Byde (2008) & $\begin{array}{l}\text { Survey envolvendo } 238 \text { empresas no } \\
\text { Reino Unido. }\end{array}$ & $\begin{array}{l}\text { Apontou um maior nível de } \\
\text { maturidade na gestão de projetos em } \\
\text { empresas relacionadas à construção } \\
\text { se comparadas a outros setores } \\
\text { (tecnologia da informação, projetos } \\
\text { estratégicos e projetos de mudança } \\
\text { organizacional). }\end{array}$ \\
\hline $\begin{array}{l}\text { 1dentificar fatores contribuintes que } \\
\text { impulsionarm a maturidade da gestão } \\
\text { de projetos. }\end{array}$ & Silveira (2008) & $\begin{array}{l}\text { Survey com } 473 \text { pessoas } \\
\text { diretamente envolvidas em gestão } \\
\text { de projetos. }\end{array}$ & $\begin{array}{l}\text { Verificados maiores níveis de } \\
\text { maturidade em empresas de origem } \\
\text { estrangeira, empresas de grande } \\
\text { porte e empresas de dependência } \\
\text { tecnológica. }\end{array}$ \\
\hline
\end{tabular}


mensuração e, em alguns casos, nas conclusões, eles auxiliam no embasamento teórico e no entendimento da natureza do fenômeno estudado.

Os trabalhos empíricos do Quadro 2 apresentam alguns resultados similares e outros conflitantes. Todavia, ao analisar seus métodos é possível dizer que seus instrumentos de pesquisa são distintos, o que dificulta análises comparativas ou longitudinais entre eles.

\section{Metodologia}

A abordagem metodológica desta pesquisa partiu de um levantamento bibliográfico sistêmico nas bases de dados Academic Search Premier (EBSCO), Web of Science (ISI), ABI/INFORM (ProQuest), Portal Domínio Público e Biblioteca Digital de Teses e Dissertações da USP, bem como nas revistas acadêmicas Project Management Journal (PMI) e International Project Management Journal (IPMA/Elsevier) (INTERNATIONAL..., 2006) na busca de estudos de duas naturezas: 1) trabalhos empíricos com a proposta de avaliar comparativamente a maturidade em gestão de projetos em diversos setores, e 2) trabalhos teóricos ou normativos que se propuseram a criticar e formular modelos de avaliação de maturidade em gestão de projetos.

Também foram pesquisados artigos e estudos empíricos, bem como dissertações e teses que abordaram o papel estratégico da tecnologia da informação, o alinhamento estratégico de Tl e os projetos de Tl, para contextualizar o tipo de projeto estudado.

Finalmente, foram revisados livros técnicos e identificados e pesquisados instrumentais no tema, a fim de complementar o levantamento.

0 instrumento de pesquisa foi desenvolvido com base no questionário do $0 \mathrm{PM} 3{ }^{\circledR}$ publicado em PMI (PROJECT..., 2008). Primeiramente foram selecionadas as duas dimensões relevantes para o estudo: maturidade da gestão de projetos e importância dos facilitadores organizacionais para a gestão de projetos. Com esta primeira seleção, foram excluídas questões relacionadas às dimensões de gestão de programas e de gestão de portfólio.

Com base na revisão bibliográfica, incluiu-se também um nível adicional na escala de maturidade, um nível embrionário ou preliminar que antecede o primeiro grau de maturidade proposto em PMI (PROJECT..., 2008). Assim, neste estudo foi adicionado na escala um nível de maturidade que precede o nível inicial proposto pelo $0 \mathrm{PM} 3^{\circledR}$, que é a padronização.

Dessa forma, o questionário foi traduzido e formatado para ficar no formato web-survey. Posteriormente, esse instrumento passou por pré-teste e foi ajustado com base nas comendações e melhorias identificadas. Seu formato e conteúdo estão parcialmente representados nas Figuras 1 e 2.

Já o trabalho de campo consistiu na busca de potenciais organizações e respondentes para comporem a amostra do fenômeno em estudo.

Para tanto, buscou-se nas redes sociais profissionais que pudessem contribuir para a pesquisa, por meio de análise de currículo e experiência publicada no Linkedln e Facebook/BranchOut, bem como fez-se uso da rede de contatos da FEA-USP e do pesquisador para envio do questionário.

Durante o levantamento de dados realizou-se uma análise individual dos questionários com o intuito de excluir da amostra aqueles que não pudessem ser considerados válidos. Assim, 468 respondentes visualizaram o questionário, 357 o responderam parcialmente, 225 responderam até a última seção, 217 questionários foram considerados válidos e, dado o enfoque do trabalho, selecionaram-se somente os

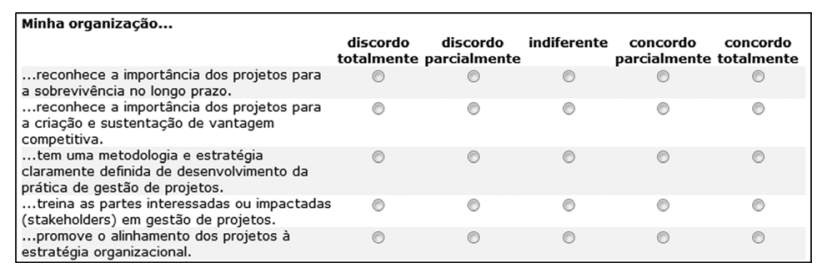

Figura 1. Facilitadores organizacionais à gestão de projetos $\left(\mathrm{OPM} 3^{\circledR}\right)$.

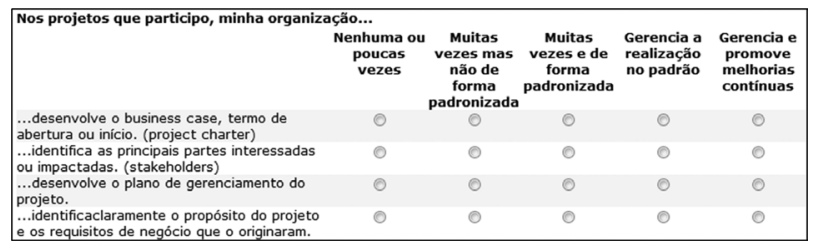

Figura 2. Maturidade em gestão de projetos (OPM3 $\left.{ }^{\circledR}\right)$. 
190 questionários oriundos de profissionais que atuam predominantemente com Tl. A redução nesse último filtro foi pequena, pois a pesquisa foi intencionalmente enviada somente aos profissionais dessa área que atuam com projetos de sistemas de informações.

Finalmente, foram realizados testes estatísticos, análise descritiva, análise estatística e análise de regressão.

O software estatístico SPSS na versão 18 foi utilizado para análise dos dados. Nas respostas válidas foi aplicado o teste de Kolmogorov-Smirnov para avaliar a normalidade das distribuições das respostas nas partes 3 e 4 do questionário, conforme ilustrado na Tabela 1.

Verificou-se que dados coletados sobre a percepção da maturidade da gestão de projetos se manifestaram como uma distribuição não normal (Sig. 0,044) com cauda longa à esquerda (negative skewness), enquanto os dados coletados sobre a percepção da importância dos facilitadores organizacionais da gestão de projetos, por sua vez, manifestaram as características de distribuição normal (Sig. 0,158).

Dadas as características das distribuições, as análises foram compostas pelo teste não paramétrico de Kruskal-Wallis. Segundo Fávero et al. (2009), o teste Kruskal-Wallis pode ser aplicado quando a normalidade e igualdade das variâncias não estejam presentes e há a probabilidade de as amostras independentes serem da mesma população.

\section{Análise de dados}

A amostra de dados mostrou-se significativa para o estudo ao se analisar de maneira descritiva tanto as características dos respondentes quanto as das empresas por eles representadas. Nas Tabelas 2 e 3 é possível avaliar algumas características para se entender melhor a representatividade da amostra.

Outras características relevantes dos respondentes apontam que $82,95 \%$ possuem curso universitário,

Tabela 1. Análise da distribuição da maturidade e dos facilitadores organizacionais.

\begin{tabular}{|c|c|c|c|}
\hline \multicolumn{2}{|c|}{ Teste de Kolmogorov-Smirnov } & \multirow{2}{*}{$\begin{array}{c}\text { Maturidade em gestão de projetos } \\
190\end{array}$} & \multirow{2}{*}{$\begin{array}{c}\text { Facilitadores organizacionais } \\
190\end{array}$} \\
\hline $\mathrm{N}$ & & & \\
\hline \multirow{2}{*}{ Parâmetros normais $(\mathrm{a}, \mathrm{b})$} & Mediana & 54,4158 & 51,7316 \\
\hline & Desvio padrão & 21,75333 & 13,69457 \\
\hline \multirow{3}{*}{ Diferença mais extrema } & Absoluta & 0,100 & 0,082 \\
\hline & Positivo & 0,100 & 0,046 \\
\hline & Negativo & $-0,066$ & $-0,082$ \\
\hline Kolmogorov-Smirnov Z & & 1,380 & 1,126 \\
\hline Sig. assint. (bicaudal) & & 0,044 & 0,158 \\
\hline
\end{tabular}

a. Distribuição testada é normal, b. Calculado a partir dos dados.

Tabela 2. Análise descritiva dos respondentes.

\begin{tabular}{|c|c|c|c|c|}
\hline & Função atual ou mais recente do respondente & $\mathrm{N}$ & $\%$ & Somatória da maturidade \\
\hline \multirow{6}{*}{ Maturidade } & Estagiário ou trainee & 4 & 2,11 & 108,75 \\
\hline & Analista ou operador & 27 & 14,21 & 74,44 \\
\hline & Líder ou coordenador de equipe & 66 & 34,37 & 95,22 \\
\hline & Gerente ou superintendente de área & 65 & 34,21 & 102,10 \\
\hline & Alta administração, diretor, VP ou c-level & 28 & 14,74 & 99,25 \\
\hline & Total & 190 & 100,00 & \\
\hline
\end{tabular}

Tabela 3. Análise descritiva das organizações.

\begin{tabular}{|c|c|c|c|c|}
\hline Faixa de faturamento ou orçamento (IBGE) & $\begin{array}{c}\text { Número de } \\
\text { funcionários (IBGE) }\end{array}$ & Somatória da maturidade & $\mathrm{N}$ & $\begin{array}{l}\text { Desvio } \\
\text { padrão }\end{array}$ \\
\hline \multirow{3}{*}{$\begin{array}{l}\text { PME (pequenas e médias), } \\
\text { menor que R\$ } 300 \text { milhões }\end{array}$} & < 499 funcionários & 50,66 & 83 & 20,74 \\
\hline & $\geq 500$ funcionários & 54,88 & 16 & 16,61 \\
\hline & Total & 51,34 & 99 & 20,11 \\
\hline \multirow{3}{*}{$\begin{array}{l}\text { Grandes organizações, } \\
\text { maior que R\$ } 300 \text { milhões }\end{array}$} & < 499 funcionários & 48,33 & 6 & 16,86 \\
\hline & $\geq 500$ funcionários & 58,67 & 83 & 23,31 \\
\hline & Total & 57,98 & 89 & 23,01 \\
\hline \multirow{3}{*}{ Total } & < 499 funcionários & 50,51 & 89 & 20,43 \\
\hline & $\geq 500$ funcionários & 58,06 & 99 & 22,34 \\
\hline & Total & 54,48 & 188 & 21,73 \\
\hline
\end{tabular}


MBA, pós-graduação ou especialização; que 83,66\% possuem mais de sete anos de experiência profissional; que 50,78\% possuem mais de sete anos de experiência profissional em projetos; e que $17,12 \%$ possuem alguma certificação em gestão de projetos.

Outros dados importantes de nosso estudo revelam que $84,71 \%$ atuam há mais de sete anos no mercado; que $57,26 \%$ são de multinacionais; e que $71,55 \%$ têm origem latino-americana, mais significativamente, brasileira.

Como se pode verificar na Tabela 4, há justificativas estatísticas que sugerem não existirem diferenças da maturidade da gestão de projetos de $\mathrm{Tl}$ entres as organizações dos setores de serviços, manufatura/ indústria, comercial/varejo, setor público e terceiro setor (Sig. 0,407).

Entretanto, ao se dividirem as organizações em dois agrupamentos distintos - sendo o primeiro composto por organizações de grande porte com faturamento anual maior que $\mathrm{R} \$ 300$ milhões e o segundo com as demais - encontraram-se evidências estatísticas para afirmar que as grandes organizações parecem ter maior maturidade em gestão de projetos de $\mathrm{Tl}$ do que as demais (Sig. 0,048), conforme Tabela 5.

Como se aferiu, ao se dividirem as mesmas organizações em dois agrupamentos distintos - o primeiro composto por grandes organizações com 500 ou mais funcionários e o segundo com as que possuíam 499 ou menos funcionários - apontaram-se evidências estatísticas para afirmar que as organizações com 500 ou mais funcionários parecem apresentar maior maturidade em gestão de projetos de $\mathrm{Tl}$ do que as que possuem menos funcionários (Sig. 0,012), como apresentado na Tabela 6.

Ao se avaliar a maturidade da gestão de projetos de $\mathrm{Tl}$ em organizações com amplitude distinta no escopo geográfico (Sig. 0,018), pôde-se afirmar que empresas de atuação internacional ou multinacional parecem apresentar maior maturidade, seguidas por empresas com atuação nacional e, por último, pelas empresas de atuação local ou regional, conforme Tabela 7.

Também parece haver certa correlação positiva da maturidade da gestão de projetos de $\mathrm{Tl}$ em relação à amplitude do escopo geográfico de atuação da organização. Assim, organizações internacionais ou multinacionais apresentaram maior maturidade que organizações regionais ou nacionais que, por sua vez, apresentaram maior maturidade que organizações locais.

Tendo em vista que a maturidade da gestão de projetos é um fenômeno com diversas nuances ou perspectivas compostas, resolveu-se avaliar de maneira distinta a maturidade por área de conhecimento. Assim, realizaram-se testes de Kruskal Wallis buscando investigar possíveis diferenças de maturidade em uma área de conhecimento em específico, ao invés de somente avaliar a maturidade de maneira geral.

Tabela 4. Maturidade por setor de atividade.

\begin{tabular}{|c|c|c|c|}
\hline $\begin{array}{l}\text { Teste de Kruskal Walliscom } \\
\text { agrupamento no setor }\end{array}$ & Setor de atividade & $\mathrm{N}$ & Ordem das medianas \\
\hline \multirow{6}{*}{ Maturidade de Gestão de Projetos de Tl } & Serviços & 133 & 97,40 \\
\hline & Comercial & 3 & 78,33 \\
\hline & Manufatura & 44 & 93,42 \\
\hline & Setor público & 8 & 63,81 \\
\hline & Terceiro setor & 1 & 144,50 \\
\hline & Total & 189 & \\
\hline Teste estatístico & Percepção da maturidade & & \\
\hline Chi-quad. & 3,993 & & \\
\hline Df & 4 & & \\
\hline Sig. Assint. & 0,407 & & \\
\hline
\end{tabular}

Tabela 5. Maturidade por agrupamento de faturamento.

\begin{tabular}{|c|c|c|c|}
\hline Teste de Kruskal Wallis & Faixa de faturamento ou orçamento (IBGE) & $\mathrm{N}$ & Ordem das medianas \\
\hline \multirow{3}{*}{ Maturidade da gestão de projetos de Tl } & $\begin{array}{l}\text { PME (pequenas e médias), } \\
\text { menor que R\$ } 300 \text { milhões }\end{array}$ & 99 & 87,49 \\
\hline & $\begin{array}{l}\text { Grandes organizações, } \\
\text { maior que } \mathrm{R} \$ 300 \text { milhões }\end{array}$ & 90 & 103,26 \\
\hline & Total & 189 & \\
\hline Teste estatístico & Percepção da maturidade & & \\
\hline Chi-quad. & 3,920 & & \\
\hline Df & 1 & & \\
\hline Sig. Assint. & 0,048 & & \\
\hline
\end{tabular}


No entanto, o resultado do teste estatístico não permite afirmar que a maturidade da gestão de projeto de $\mathrm{Tl}$ de cada uma das nove áreas de conhecimento é diferente de um setor de atividade para outro considerando-se o nível de confiança de 95\% (Sig. > 0,050). Mesmo ao relaxarmos o nível de confiança para 90\% ainda não foram encontradas diferenças estatisticamente significativas (Sig. > 0,100).
Dessa forma não foram encontradas diferenças estatísticas significativas da maturidade de gestão de projetos de $\mathrm{Tl}$ entre um setor e outro, o que se procurou resumir na Tabela 8.

Embora não tenham sido encontradas diferenças estatisticamente significativas, plotou-se em formato de radar os resultados das medianas de maturidade da gestão de projetos de tecnologia de informação para

Tabela 6. Maturidade por agrupamento de quantidade de funcionários.

\begin{tabular}{|c|c|c|c|}
\hline Teste de Kruskal Wallis & Quantidade de funcionários (agrup.) & $\mathrm{N}$ & $\begin{array}{c}\text { Ordem das } \\
\text { medianas }\end{array}$ \\
\hline & de 0 a 499 funcionários & 90 & 84,93 \\
\hline \multirow[t]{2}{*}{ Maturidade da gestão de projetos de $\mathrm{Tl}$} & 500 funcionários ou mais & 100 & 105,02 \\
\hline & Total & 190 & \\
\hline Teste estatístico & Percepção da maturidade & & \\
\hline Chi-quad. & 6,323 & & \\
\hline Df & 1 & & \\
\hline Sig. Assint. & 0,012 & & \\
\hline
\end{tabular}

Tabela 7. Maturidade por escopo geográfico.

\begin{tabular}{|c|c|c|c|}
\hline Teste de Kruskal Wallis & Escopo geográfico (amplitude) da organização & $\mathrm{N}$ & $\begin{array}{c}\text { Ordem das } \\
\text { medianas }\end{array}$ \\
\hline \multirow{4}{*}{ Maturidade da gestão de projetos } & Local e/ou regional (um ou mais municípios/estados) & 29 & 77,28 \\
\hline & Nacional (duas ou mais regiões) & 47 & 83,28 \\
\hline & Internacional ou multinacional (dois ou mais países) & 112 & 103,67 \\
\hline & Total & 188 & \\
\hline Teste estatístico & Percepção de maturidade & & \\
\hline Chi-quad. & 8,089 & & \\
\hline Df & 2 & & \\
\hline Sig. Assint. & 0,018 & & \\
\hline
\end{tabular}

Tabela 8. Mediana da maturidade da gestão de projeto por setor de atividade e área do conhecimento.

\begin{tabular}{|c|c|c|c|c|c|c|c|c|c|c|}
\hline \multicolumn{2}{|c|}{ Setor de atividade } & $\begin{array}{c}\text { Integra- } \\
\text { ção }\end{array}$ & Escopo & Tempo & Custo & Qualidade & $\mathrm{RH}$ & $\begin{array}{l}\text { Comuni- } \\
\text { cações }\end{array}$ & Riscos & $\begin{array}{l}\text { Aquisi- } \\
\text { ções }\end{array}$ \\
\hline \multirow{3}{*}{ Serviços } & Mediana & 2,94 & 2,93 & 3,41 & 3,35 & 2,83 & 2,70 & 2,56 & 2,63 & 2,86 \\
\hline & $\mathrm{N}$ & 133 & 133 & 133 & 133 & 133 & 133 & 133 & 133 & 133 \\
\hline & Desv. padrão & 1,24 & 1,22 & 1,13 & 1,21 & 1,35 & 1,33 & 1,35 & 1,34 & 1,28 \\
\hline \multirow{3}{*}{ Comercial } & Mediana & 2,89 & 2,67 & 2,78 & 2,67 & 2,67 & 3,00 & 2,67 & 2,60 & 2,67 \\
\hline & $\mathrm{N}$ & 3 & 3 & 3 & 3 & 3 & 3 & 3 & 3 & 3 \\
\hline & Desv. padrão & 1,84 & 2,03 & 1,95 & 2,08 & 2,08 & 1,73 & 2,08 & 2,08 & 2,08 \\
\hline \multirow{3}{*}{ Manufatura } & Mediana & 2,93 & 2,75 & 3,25 & 3,32 & 2,75 & 2,50 & 2,45 & 2,50 & 3,16 \\
\hline & $\mathrm{N}$ & 44 & 44 & 44 & 44 & 44 & 44 & 44 & 44 & 44 \\
\hline & Desv. padrão & 1,17 & 1,27 & 1,25 & 1,34 & 1,45 & 1,41 & 1,41 & 1,27 & 1,27 \\
\hline \multirow{3}{*}{ Setor público } & Mediana & 2,08 & 2,00 & 2,42 & 2,50 & 2,00 & 2,00 & 2,13 & 2,10 & 2,38 \\
\hline & $\mathrm{N}$ & 8 & 8 & 8 & 8 & 8 & 8 & 8 & 8 & 8 \\
\hline & Desv. padrão & 0,89 & 1,15 & 1,07 & 1,51 & 0,93 & 0,93 & 0,83 & 0,78 & 1,06 \\
\hline \multirow{3}{*}{ Terceiro setor } & Mediana & 4,33 & 2,67 & 5,00 & 5,00 & 5,00 & 3,00 & 3,00 & 3,20 & 5,00 \\
\hline & $\mathrm{N}$ & 1 & 1 & 1 & 1 & 1 & 1 & 1 & 1 & 1 \\
\hline & Desv. padrão & - & - & - & - & - & - & - & - & - \\
\hline \multirow{3}{*}{ Total } & Mediana & 2,91 & 2,85 & 3,33 & 3,30 & 2,79 & 2,63 & 2,52 & 2,58 & 2,92 \\
\hline & $\mathrm{N}$ & 189 & 189 & 189 & 189 & 189 & 189 & 189 & 189 & 189 \\
\hline & Desv. padrão & 1,23 & 1,24 & 1,18 & 1,28 & 1,38 & 1,33 & 1,35 & 1,31 & 1,29 \\
\hline
\end{tabular}



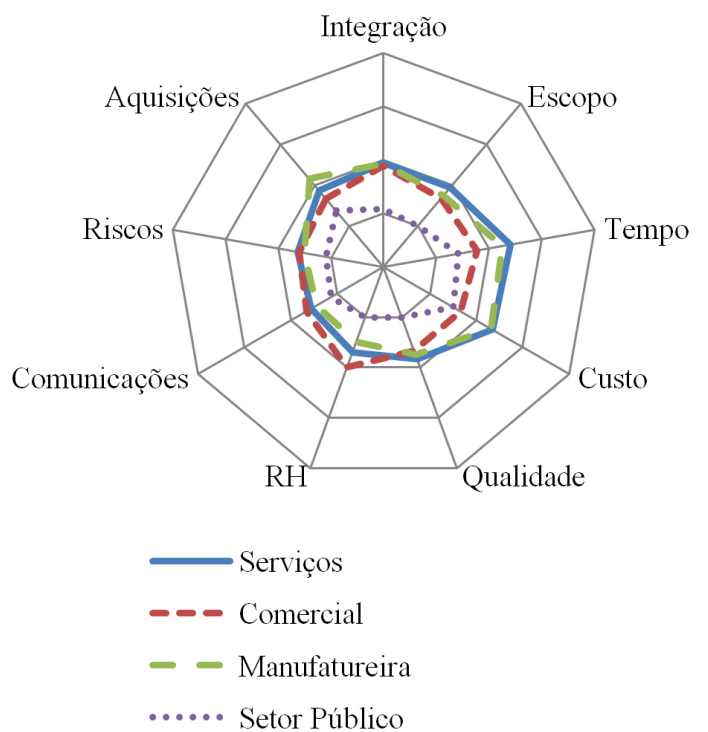

Figura 3. Mediana de maturidade das áreas de conhecimento por setor de atividade.

estudos futuros e para demonstrar que, graficamente, é possível identificar diferenças mesmo que elas não sejam estatisticamente significativas (Figura 3).

\section{Considerações finais}

Primeiramente, ao realizar o teste KolmogorovSmirnov para avaliar a normalidade da distribuição da percepção de maturidade, foi identificada uma distribuição não normal, o que pode sinalizar que o uso de médias não seria o mais apropriado para retratar o fenômeno, conforme foi utilizado em outros estudos empíricos anteriores.

Dessa forma, os trabalhos de Levene, Bentley e Jarvis (1995), Kwak e lbbs (2000a, b), Cooke-Davies e Arzymanow (2003), Crawford (2005), Mullaly (2006), Zwikael e Globerson (2006) e Byde (2008) poderiam ser questionados quanto à simples comparação de médias de maturidade como fator determinante para afirmar que um setor ou outro tem maior maturidade em gestão de projetos. Esses trabalhos comparam a maturidade da gestão de projetos de diversos setores de maneira geral, ou seja, sem analisar as maturidades por categoria de projeto (ARCHIBALD, 2004).

A comparação multissetorial sem categoria definida pode ser inadequada, uma vez que ela compara a maturidade de projetos de tipos distintos entre si. Assim, cabe reiterar que não foram encontradas diferenças estatísticas significativas de maturidade de gestão de projetos de $\mathrm{Tl}$ entre um setor ou outro.

No entanto, os dados do presente estudo sugerem que a maturidade da gestão de projetos está longe de ser uma commodity, mesmo dentro do mesmo setor. Dessa forma, os modelos de maturidade não podem ser considerados como as "balas de prata" da vantagem competitiva (JUGDEV; THOMAS, 2002).

Pode-se assim sugerir que desenvolver a maturidade em gestão de projetos é somente parte da equação, sendo que complementarmente se deve buscar alavancar o resultado e os fatores críticos de sucesso dos projetos (FORTUNE; WHITE, 2006).

Adicionalmente, os dados coletados não mostram evidências de que a maturidade da gestão de projetos de Tl está relacionada com o setor de atividade, como identificado em Levene, Bentley e Jarvis (1995). De qualquer forma, a análise de dados sugere que a maturidade da gestão de projetos de Tl parece ser maior em grandes organizações que possuem faturamento superior a $\mathrm{R} \$ 300$ milhões ou têm mais de 500 funcionários.

Também parece haver certa correlação entre a maturidade da gestão de projetos de Tl e a amplitude de atuação da organização em termos de escopo geográfico. Os dados coletados parecem sugerir que organizações internacionais ou multinacionais apresentam maior maturidade que organizações regionais ou nacionais que, por sua vez, apresentam maior maturidade que organizações locais.

Embora nos últimos anos muitas empresas estejam adotando a gestão de portfólio como ferramenta de alinhamento estratégico dos projetos, os dados parecem indicar que esse processo formalizado e estruturado é mais presente nas grandes organizações, o que pode ser explicado pela dificuldade de monitoramento e alinhamento do portfólio de projetos em contextos mais complexos.

Contudo, o papel estratégico dos projetos e a real disponibilização de recursos para a execução dos projetos ainda são pouco valorizados em empresas de pequeno e médio porte ou de atuação local/regional.

Questiona-se também, com base em Blichfeldt e Eskerod (2008), uma possível lacuna nos modelos normativos de gestão de portfólio de projetos para lidar com os projetos extraoficiais. A estratégia emergente sinaliza a existência de um processo muito menos deliberado e que muitas vezes é derivado da própria execução e implementação da estratégia oficial (MINTZBERG, 1994; COLLINS, 2001).

Explorando-se assim as fronteiras da gestão de projetos de $\mathrm{Tl}$ em direção à gestão de projetos em geral poder-se-ia dizer que avaliar a maturidade da gestão de projetos com o objetivo de melhorá-la é a maneira pela qual as organizações buscam uma execução mais eficiente dos projetos a fim de obter melhores taxa de sucesso. 
São limitações do presente estudo a amostra por conveniência, concentrada em grandes empresas nos setores de serviços e manufatura; sendo assim, as interpretações são mais seguras na análise desses setores.

Também é limitação da pesquisa que sustenta este artigo o método sobre aplicação de questionários que avaliou de maneira indireta a maturidade de projetos. A percepção dos respondentes sobre a maturidade de projetos, considerando os projetos nos quais eles atuaram, de alguma forma pode eventualmente gerar distorções de interpretação tanto por parte de quem está sendo pesquisado quanto por parte daquele que pesquisa.

Sugere-se que novos trabalhos investiguem a maturidade na gestão de projetos, introduzindo as práticas de execução, monitoramento, controle e encerramento.

Também se propõe que estudos posteriores investiguem as práticas e a maturidade na gestão de projetos dentro do mesmo setor e ou entre setores distintos, comparando-as entre os projetos de diferentes categorias de Archibald (2004) - P\&D, Tl, operações/produção -, além de pesquisarem possíveis diferenças entre as práticas e a maturidade da gestão de projetos corporativos (internos) e os projetos realizados sob contrato (externos), em empresas baseadas em projetos.

\section{Referências}

ARCHIBALD, R. D. A global system for categorizing projects: the need for, recommended approach to, and practical uses of the system. In: LATIN AMERICAN FORUM: PM IN GOVERNMENT, 2., 2004, Brasilia. Anais... Brasília, 2004.

$\mathrm{BAl}$, S. et al. Organizational project selection based on fuzzy multi-index evaluation and bp neural network. In: INTERNATIONAL CONFERENCE ON MANAGEMENT AND SERVICE SCIENCE, 2010, Massachusetts. Proceedings... IEEE, 2010.

BARNEY, J. B.; HESTERLY, W. S. Administração estratégica e vantagem competitiva. Pearson Prentice Hall,1986.

BERSSANETI, F. T. et al. Maturity and performance in project management: a survey of information technology professional. In: POMS ANNUAL CONFERENCE, 19., 2008, La Jolla. Proceedings... La Jolla, 2008.

BLICHFELDT, B. S.; ESKEROD, P. Project portfolio management - there's more to it than what management enacts. International Journal of Project Management, v. 26, p. 357-365, 2008. http://dx.doi.org/10.1016/j. ijproman.2007.06.004

BYDE, D. J. Is construction different? A comparison of perceptions of project management performance and practices by business sector and project type. Construction Management and Economics, n. 26, 2008.

CHANDLER, A. D. Strategy and structure. Cambridge: MIT Press, 1962.
COLLINS, J. Good to great: why some companies make the leap and other's don't. New York: Harper Business, 2001.

COLLINS, J. How the mighty fall: and why some companies never give in. New York: Harper Business, 2009.

COLlINS, J.; HANSEN, M. T. Great by choice: uncertainty, chaos, and luck - why some thrive despite them all. New York: Harper Business. 2011.

COOKE-DAVIES, T. J.; ARZYMANOW, A. The maturity of project management in different industries: an investigation into variations between project management models. International Journal of Project Management, n. 21, p. 471-478. 2003. http://dx.doi.org/10.1016/ S0263-7863(02)00084-4

COOPER, R.; EDGETT, S.; KLEINSCHMIDT, E. Portfolio management - fundamental to new product success. Product Development Institute, 2001a. Working Paper n. 12.

COOPER, R.; EDGETT, S.; KLEINSCHMIDT, E. Portfolio management of new product development: results of an industry. Practices, study. $R \& D$ Management, n. 31, $2001 \mathrm{~b}$.

CRAWFORD, L. Senior management perceptions of project management competence. International Journal of Project Management, n. 23, 2005.

CRAWFORD, L.; HOBBS, J. B.; TURNER, J. R. Project categorization systems: aligning capability with strategy for better results. Newtown Square: Project Management Institute, 2004.

DEMING, W. E. Out of the crisis. Massachusetts: The MIT Press, 1986.

DORLING, A. SPICE: Software process improvement and capability determination. Software Quality Journal, v. 2, n. 4, p. 209-224, 1993. http://dx.doi.org/10.1007/ BF00403764

FÁVERO, L. P. et al. Análise de dados: modelagem multivariada para tomada de decisões. Rio de Janeiro: Elsevier, 2009.

FLYVBJERG, B.; BUDZIER, A. Why your it project may be riskier than you think. Harvard Business Review, 2011.

FORTUNE, J.; WHITE, D. Framing of project critical success factors by a systems model. International Journal of Project Management, n. 24. 2006.

FRAME, J. D. Project management competence: building key skills for individuals, teams and organizations. San Francisco: Josey-Bass, 1999.

GRANT, K. P.; PENNYPACKER, J. S. Project management maturity: an assessment of project management capabilities among and between selected industries. IEEE Transactions of Engineering Management, v. 53, n. 1, 2006. http://dx.doi.org/10.1109/TEM.2005.861802

GUANGSHE, J. et al. Application of Organizational Project Management Maturity Model (OPM3) to construction in China: an empirical study. In: INTERNATIONAL CONFERENCE ON INFORMATION MANAGEMENT INNOVATION MANAGEMENT AND INDUSTRIAL ENGINEERING, 2008, Taipei. Proceedings... IEEE, 2008. v. 2, p. 56-62.

GUEDES, R. M. et al. Alinhamento do portfólio de projetos à estratégia das organizações: um estudo exploratório quantitativo. E\&G - Revista Economia e Gestão da PUC Minas, v. 11, n. 27, p. 67-93, 2011.

HAIRE, M. Modern organizational theory. New York: John Wiley, 1959. 
HARTER, D. E.; KRISHNAN, M. S.; SLAUGHTER, S. A. Effects of process maturity on quality, cycle time, and effort in software product development. Management Science, v. 46, n. 4, p. 451-466, 2000. http://dx.doi.org/10.1287/ mnsc.46.4.451.12056

IBBS, W. C.; REGINATTO, J.; KWAK, Y. H. Developing project management capability: benchmarking, maturity, modeling, gap analysis and ROI studies. In: MORRIS, P. W. G.; PINTO, J. K. The wiley guide to managing projects. New Jersey: John Wiley, 2004.

INTERNATIONAL PROJECT MANAGEMENT ASSOCIATION - IPMA. IPMA Competence Baseline, Version 3.0. Nijkerk, 2006.

JEFFERY, M.; LELIVELD, 1. Best practices in it portfolio management. Massachusetts: MIT Sloan Management Review, 2004.

JUGDEV, K.; THOMAS, J. Project management maturity models: the silver bullets of competitive advantage? Project Management Journal, v. 33, n. 4, p. 2-14, 2002.

KANUP, W. R. Modelos de maturidade: um caminho para as organizações alcançarem maturidade em gerenciamento de projetos como diferencial estratégico. 2009. Dissertação (Mestrado)-Pontifícia Universidade Católica de São Paulo, São Paulo, 2009.

KERZNER, H. Strategic planning for project management: using a project management maturity model. New York: John Wiley, 2001.

KWAK, Y. H.; IBBS, C. W. Assessing project management maturity. Project Management Journal, v. 31, n. 1, p. 3243, 2000a.

KWAK, Y. H.; IBBS, C. W. Calculating project management's return on investment. Project Management Journal, v. 31 , n. 2 , p. $38-47,2000$ b.

LAURINDO, F. J. B. Tecnologia da informação: planejamento e gestão de estratégias. São Paulo: Atlas, 382. p. 2008.

LEE, L. S.; ANDERSON, R. M. An exploratory investigation of the antecedents of the it project management capability. e-Service Journal, 2006. http://dx.doi.org/10.2979/ ESJ.2006.5.1.27

LEVENE, R. J.; BENTLEY, A. E.; LARVIS, G. S. The scale of project management. In: ANNUAL PROJECT MANAGEMENT INSTITUTE SEMINAR \& SYMPOSIUM, 26., 1995. Proceedings... PMI, 1995.

LUFTMAN, J.; KEMPAIAH, R. M. The IS organization of the future: the it talent challenge. Information Systems Management, v. 24, p. 129-138, 2007. http://dx.doi. org/10.1080/10580530701221023

LUKOSEVICIUS, A. P. Maturidade em gerenciamento de projetos e desempenho de projetos na indústria naval brasileira de construção de plataformas de petróleo flutuantes. 2005. Dissertação (Mestrado)-Faculdades IBMEC, Rio de Janeiro, 2005.

MAXIMIANO, A. C. A.; RABECHINI, R. Maturidade em gestão de projetos: análise de um caso e proposição de um modelo. In: SIMPÓSIO DE GESTÃO DA INOVAÇÃO TECNOLÓGICA, 2002, Salvador. Anais... Salvador, 2002.

McFARLAN, F. W. Portfolio Approach to Information Systems. Harvard Business Review. Boston, p. 142-150, 1981

MICHAELIS. Moderno dicionário da língua portuguesa. São Paulo: Melhoramentos, 1998.

MINTZBERG, H. Rise and fall of strategic planning. New York: The Free, 1994.
MOORE, G. A. Inside the tornado: strategies for developing, leveraging, and surviving hypergrowth markets. Harper Business, 2004.

MOORE, G. A. Living on the fault line: managing for shareholder value in any economy. 2nd ed. Harper Business, 2002.

MORAES, R. 0.; KRUGLIANSKAS, 1. Projetos de T1: maturidade $\mathrm{x}$ desempenho. RAl - Revista de Administração e Inovação, v. 7, n. 2, p. 22-33, 2010.

MULLALY, M. Longitudinal analysis of project management maturity. Project Management Journal, v. 36, n. 3, p. 6273, 2006.

PORTER, M. E. Competitive strategy: techniques for analyzing industries and competitors. New York: The Free Press, 1980.

PRADO, D. MMGP: um modelo brasileiro de maturidade em gerenciamento de projetos. Ponto GP, 2006. Disponivel em: <http://pontogp.wordpress.com/2006/05/06/ mmgp-um-modelo-brasileiro-de-maturidade-emgerenciamento-de-projetos/>. Acesso em: 10 jul. 2010.

PRAD0, D. Maturidade em gerenciamento de projetos. Nova Lima: INDG Tec S, 2008a.

PRADO, D. Maturidade em gerenciamento de projetos. Revista Mundo PM, 2008b. Seção: Noticias e o Mercado. Disponivel em: <http://www.mundopm.com.br/noticia. jsp?id=259>. Acesso em: 11 jul. 2010.

PRAHALAD, C. K.; HAMEL, G. The core competence of the corporation. Harvard Business Review, 1990.

PROJECT MANAGEMENT INSTITUTE - PMI. Organizational Project Management Maturity Model (OPM3). 2nd ed. Pennsylvania: Project Management Institute, 2008.

RABECHINI JUNIOR, R. Competências e maturidade em gestão de projetos: uma perspectiva estruturada. 2003. Tese (Doutorado)-Escola Politécnica, Universidade de São Paulo, 2003.

RIVARD, S.; DUPRÉ, R. Information systems project management in PMJ: a brief history. Project Management Journal, v. 40, n. 4, p. 20-30, 2009. http://dx.doi. org/10.1002/pmj.20143

RODRIGUES, 1.; RABECHINI JUNIOR, R.; CSILLAG, J. M. Os escritórios de projetos como indutores de maturidade em gestão de projetos. RAUSP - Revista de Administração da Universidade de São Paulo, v. 41, n. 3, p. 273-287, 2006.

SALVIANO, C. F.; FIGUEIREDO, A. M. C. M. Unified basic concepts for process capability models. In: INTERNATIONAL CONFERENCE ON SOFTWARE ENGINEERING AND KNOWLEDGE ENGINEERING, 20., 2008. Proceedings... 2008. p. 173-178.

SCOTT, B. R. Stages of corporate development. Boston: Harvard Business School, Intercollegiate Case Clearing House. 1971.

SHENHAR, A. J.; WIDEMAN, R. M. Project management: from genesis to content to classification. In: OPERATIONS RESEARCH AND MANAGEMENT SCIENCE (INFORMS), 1996, Washington. Proceedings... Washington... 1996.

SHENHAR, A.; DVIR, D. Reinventando gerenciamento de projetos: a abordagem diamante ao crescimento e inovação bem-sucedidos. São Paulo: Makron Books, 2010.

SILVA JUNIOR, S. D. Relações entre os fatores críticos para a maturidade em gerenciamento de projetos e a gestão 
estratégica organizacional. 2009. Dissertação (Mestrado)Pontifícia Universidade Católica do Rio Grande do Sul, Porto Alegre. 2009.

SILVEIRA, G. A. Fatores contribuintes para a maturidade em gerenciamento de projeto: um estudo em empresas brasileiras. 2008. Tese (Doutorado)-Universidade de São Paulo, São Paulo, 2008.

SMITH, K. G.; MITCHELL, T. R.; SUMMER, C. E. Top level management priorities in diferente stages of the organizational life cycle. Academy of Management Journal, v. 28, n. 4, p. 799-821, 1985. http://dx.doi. org $/ 10.2307 / 256238$

STANDISH GROUP INTERNATIONAL. Chaos Summary 2009: 10 Laws of CHAOS. Technical Report, 2009.
VON WANGENHEIM, C. G. et al. Systematic literature review of software process capability/maturity models. In: INTERNATIONAL CONFERENCE ON SOFTWARE PROCESS IMPROVEMENT AND CAPABILITY DETERMINIATION - SPICE, 2010, Pisa. Procedings... Pisa, 2010.

YOUKER, R. The difference between different types of projects. In: PROJECT MANAGEMENT INSTITUTE CONGRESS, 1999, Philadelphia. Proceedings... Newtown Square: Project Management Institute, 1999.

ZWIKAEL, 0.; GLOBERSON, S. Benchmarking of project planning and success in selected industries. Benchmarking: An International Journal, v. 13, n. 6, 2006.

\title{
Maturity of project management information systems an exploratory quantitative study in Brazil
}

\begin{abstract}
This study is the consolidated result of a cross-industry analysis of the maturity of information systems (IT/IS) project management. A web-based survey was used to map IT/IS project management maturity through the perceptions of professionals in several organizations in Brazil. The project management maturity of 56 organizations in Brazil, grouped by industries such as services, manufacturing, retail, the public sector and not-for-profit, was analyzed. The field research was undertaken in 2011, using the results of several empirical studies and PMl's $0 \mathrm{PM} 3^{\circledR}$ framework. The paper shows that there is no evidence that overall IT/IS project maturity (in all knowledge areas) is related to the industry to which an organization belongs. On the other hand, the data provides non-parametric statistic evidence that large organizations have higher levels of overall IT/IS project management maturity.
\end{abstract}

Keywords

Project Management. Information Technology. Information Systems. Project Management Maturity. IT Projects. 